\title{
Cu-metalated carbyne acting as a promising molecular wire
}

Xingchen Tu, Hao Wang, Ziyong Shen, Yongfeng Wang, Stefano Sanvito, and Shimin Hou

Citation: The Journal of Chemical Physics 145, 244702 (2016);

View online: https://doi.org/10.1063/1.4972867

View Table of Contents: http://aip.scitation.org/toc/jcp/145/24

Published by the American Institute of Physics

\section{Articles you may be interested in}

On the tautomerisation of porphycene on copper (111): Finding the subtle balance between van der Waals interactions and hybridisation

The Journal of Chemical Physics 145, 244701 (2016); 10.1063/1.4972213

Si clusters are more metallic than bulk $\mathrm{Si}$

The Journal of Chemical Physics 145, 244302 (2016); 10.1063/1.4972813

Communication: Interaction of $\mathrm{BrO}$ radical with the surface of water

The Journal of Chemical Physics 145, 241102 (2016); 10.1063/1.4973242

The low-bias conducting mechanism of single-molecule junctions constructed with methylsulfide linker groups and gold electrodes

The Journal of Chemical Physics 147, 054702 (2017); 10.1063/1.4996745

A power series revisit of the PBE exchange density-functional approximation: The PBEpow model

The Journal of Chemical Physics 145, 244102 (2016); 10.1063/1.4972815

Effective scheme for partitioning covalent bonds in density-functional embedding theory: From molecules to extended covalent systems

The Journal of Chemical Physics 145, 244103 (2016); 10.1063/1.4972012

\section{AIP $\left.\right|^{\text {The Journal of }}$ AIP $\mid$ Chemical Physics} PERSPECTIVES 


\title{
Cu-metalated carbyne acting as a promising molecular wire
}

\author{
Xingchen Tu, ${ }^{1}$ Hao Wang, ${ }^{1}$ Ziyong Shen, ${ }^{1}$ Yongfeng Wang,,${ }^{1,2}$ Stefano Sanvito, ${ }^{3}$ \\ and Shimin $\mathrm{Hou}^{1,2, a)}$ \\ ${ }^{1}$ Centre for Nanoscale Science and Technology, Key Laboratory for the Physics and Chemistry of Nanodevices, \\ Department of Electronics, Peking University, Beijing 100871, China \\ ${ }^{2}$ Beida Information Research (BIR), Tianjin 300457, China \\ ${ }^{3}$ School of Physics, AMBER and CRANN Institute, Trinity College, Dublin 2, Ireland
}

(Received 14 October 2016; accepted 8 December 2016; published online 23 December 2016)

\begin{abstract}
The atomic structure and electronic transport properties of $\mathrm{Cu}$-metalated carbyne are investigated by using the non-equilibrium Green's function formalism combined with density functional theory. Our calculations show that the incorporation of $\mathrm{Cu}$ atom in carbyne improves its robustness against Peierls distortion, thus to make $\mathrm{Cu}$-metalated carbyne behave as a one-dimensional metal. When a finite $\mathrm{Cu}$-metalated carbyne chain is connected to two (111)-oriented platinum electrodes, nearly linear current-voltage characteristics are obtained for both the atop and adatom binding sites. This is due to the efficient electronic coupling between the $\mathrm{Cu}$-metalated carbyne chain and the Pt electrodes, demonstrating the promising applications of $\mathrm{Cu}$-metalated carbyne chains as molecular wires in future electronic devices. Published by AIP Publishing. [http://dx.doi.org/10.1063/1.4972867]
\end{abstract}

\section{INTRODUCTION}

The past decade has witnessed the glorious achievements made in the field of carbon-based nanoelectronics, which is regarded as one of the most promising technologies capable of extending Moore's law. ${ }^{1}$ Representative carbon-based nanomaterials, such as carbon nanotubes and graphene, have been heavily studied by both theorists and experimentalists, and significant scientific and industrial applications have also been exploited..$^{2-4}$ In contrast, carbyne, another member of the carbon family, has made very limited progress ${ }^{5-8}$ though many interesting physical applications have been suggested in theory. ${ }^{9-11}$ This is closely related to its atomic and electronic structures. The infinite sp-hybridized carbyne can have two different structures: cumulene with equalized double bonds and polyyne with alternating triple and single bonds. Cumulene is a metal with a half-filled conduction band, while polyyne is a semiconductor with a finite band gap caused by the bond length alternation. Although the Peierls distortion makes the polyynic structure being at the minimum of the potential energy and stretching enhances the Peierls transition, ${ }^{12}$ zeropoint atomic vibrations eliminate the distortion and restore the cumulenic structure. ${ }^{13}$ Electrical transport measurements of finite monatomic carbon chains have confirmed that the measurement conditions, including strain, have a decisive influence on their conductivity. ${ }^{14,15}$ Furthermore, even when an ohmic behavior is observed in unstrained carbon chains, the measured conductivity is still much lower than the predicted theoretical value, illustrating the key role of the contacting leads. ${ }^{15}$ Recently, Cu-metalated carbyne was synthesized on the $\mathrm{Cu}(110)$ surface under ultrahigh vacuum conditions. ${ }^{16}$ The introduction of metal atoms allows the regulation of the

\footnotetext{
a) Author to whom correspondence should be addressed. Electronic mail: smhou@pku.edu.cn
}

physical and chemical properties of carbyne itself, ${ }^{5,17-19}$ which will provide more potential utilities in nano/molecular electronics. Considering the fact that only the geometric structure and the density of states (DOS) of Cu-metalated carbyne on the $\mathrm{Cu}(110)$ surface were investigated using density functional theory (DFT) calculations, ${ }^{16}$ more detailed studies on the atomic structure and electronic transport properties of free-standing $\mathrm{Cu}$-metalated carbyne are highly desirable in order to facilitate its applications in nano/molecular electronic devices.

Here we investigate the structural and electronic transport properties of $\mathrm{Cu}$-metalated carbyne monatomic chains by employing the non-equilibrium Green's function formalism combined with density functional theory (that is, the so-called NEGF + DFT approach). ${ }^{20-29}$ Our calculations show that the incorporated $\mathrm{Cu}$ atom does not alter significantly the $\mathrm{C}-\mathrm{C}$ bond length but improves the robustness of $\mathrm{Cu}$-metalated carbyne to the electron-phonon perturbations and thus makes it an ideal one-dimensional metal. A finite $\mathrm{Cu}$-metalated carbyne chain connected to two $\mathrm{Cu}$ electrodes does not conduct well because of the symmetry mismatch between the $\pi$-type energy bands of the molecule and the s-type bands of bulk copper around the Fermi level. However, the transmission is much enhanced and the current increases linearly following the increase of the applied bias when platinum electrodes are used. This illustrates the intrinsic high conductive capacity of $\mathrm{Cu}$-metalated carbyne and the vital role of the electronic coupling at the molecule-electrode interfaces in determining the transport properties.

\section{CALCULATION METHOD}

In this work we use the SIESTA code ${ }^{30}$ to compute the atomic structure of $\mathrm{Cu}$-metalated carbyne chains and the quantum transport code SMEAGOL ${ }^{27-29}$ to study their electronic transport properties when contacted with $\mathrm{Cu}$ and $\mathrm{Pt}$ electrodes. 
SIESTA is an efficient DFT package, which makes use of improved Troullier-Martins pseudopotentials for describing the atomic cores and adopts a finite-range numerical orbital basis set to expand the wave functions of the valence electrons..$^{20,31}$ While a double-zeta plus polarization (DZP) basis set is used for $\mathrm{C}$ and $\mathrm{Cu}$, two different types of basis functions are used for Pt, respectively, in the bulk and at the surface. In more detail, a DZP basis is used for the Pt surface atoms, while a single-zeta plus polarization (SZP) basis is used for the bulk. The exchange-correlation functional is at the level of the generalized gradient approximation (GGA) within the Perdew-Burke-Ernzerhof (PBE) formulation. ${ }^{32}$ Geometry optimization is performed by standard conjugate gradient relaxation until the atomic forces are smaller than $0.02 \mathrm{eV}^{-1}$.

SMEAGOL is a practical implementation of the NEGF + DFT approach, which employs SIESTA as the DFT platform. ${ }^{27-29}$ We use an equivalent cutoff of 250.0 Ry for the real space grid. The charge density is integrated over 16 energy points along the semi-circle, 16 along the line in the complex plane, 400 along the real axis, while 16 poles are used for the Fermi function (the electronic temperature is $300 \mathrm{~K}$ ). The convergence criterion for the density matrix is set to be $1 \times 10^{-4}$. We always consider periodic boundary conditions in the plane transverse to the transport. The unit cell of the extended molecule comprises a finite $\mathrm{Cu}$-metalated carbyne chain and ten $\mathrm{Cu}(111)$ or $\mathrm{Pt}(111)$ atomic layers with a $(3 \times 3)$ in plane supercell. The current-voltage $(I-V)$ characteristics of the junction is calculated as

$$
I=\frac{2 e}{h} \int_{-\infty}^{+\infty} T(V, E)\left[f\left(E-\mu_{L}\right)-f\left(E-\mu_{R}\right)\right] d E,
$$

where $T(V, E)$ is the bias-dependent transmission coefficient of the junction, $f(E)$ is the Fermi function, and $\mu_{\mathrm{L} / \mathrm{R}}=E_{\mathrm{F}} \pm e V / 2$ is the local Fermi level of the left/right electrode with $E_{\mathrm{F}}$ being the Fermi energy. Then, the total transmission coefficient $T(V, E)$ of the junction is evaluated as

$$
T(V, E)=\frac{1}{\Omega_{2 D B Z}} \int_{2 D B Z} T(\vec{k} ; V, E) d \vec{k},
$$

where $\Omega_{2 \mathrm{DBZ}}$ is the area of the two-dimensional Brillouin zone (2DBZ) in the transverse directions. The $k$-dependent transmission coefficient $T(\vec{k} ; V, E)$ is obtained as

$$
T(\vec{k} ; V, E)=\operatorname{Tr}\left[\Gamma_{L} G_{M}^{R} \Gamma_{R} G_{M}^{R+}\right],
$$

where $G_{M}^{R}$ is the retarded Green's function matrix of the extended molecule and $\Gamma_{\mathrm{L}}\left(\Gamma_{\mathrm{R}}\right)$ is the broadening function matrix describing the interaction of the extended molecule with the left-hand (right-hand) side electrode. Here, we calculate the transmission coefficient by sampling $11 \times 11$ $(3 \times 3) \mathrm{k}$-points in the transverse $2 \mathrm{DBZ}$ when $\mathrm{Pt}(\mathrm{Cu})$ is used as the electrode material.

\section{RESULTS AND DISCUSSION}

We start our studies from the investigation of the atomic and electronic structures of an infinite free-standing $\mathrm{Cu}-$ metalated carbyne monatomic chain. After geometry optimization, the lattice vector of the $\mathrm{Cu}$-metalated carbyne monatomic chain is determined to be $\mathrm{a}=4.93 \AA$ with a $\mathrm{C}-\mathrm{C}$ bond length of $1.29 \AA$ and a $\mathrm{C}-\mathrm{Cu}$ bond length of $1.82 \AA$ (Fig. 1(a)). These are both slightly shorter than those in the $\mathrm{Cu}$-metalated carbyne chain on $\mathrm{Cu}(110) .{ }^{16}$ It should be noted that the optimized $\mathrm{C}-\mathrm{C}$ bond length in the $\mathrm{Cu}$-metalated carbyne chain is almost the same as that in the clean cumulene chain calculated at the same level (Fig. 1(b)). In contrast, the metalated $\mathrm{Cu}$ atoms significantly modify the band structure of the cumulene chain. For the clean cumulene chain placed along the z-axis, there is only one filled energy band below the Fermi level which is composed of the carbon $2 s$ and $2 p_{z}$ atomic orbitals. Furthermore, there are two degenerate $\pi$-type
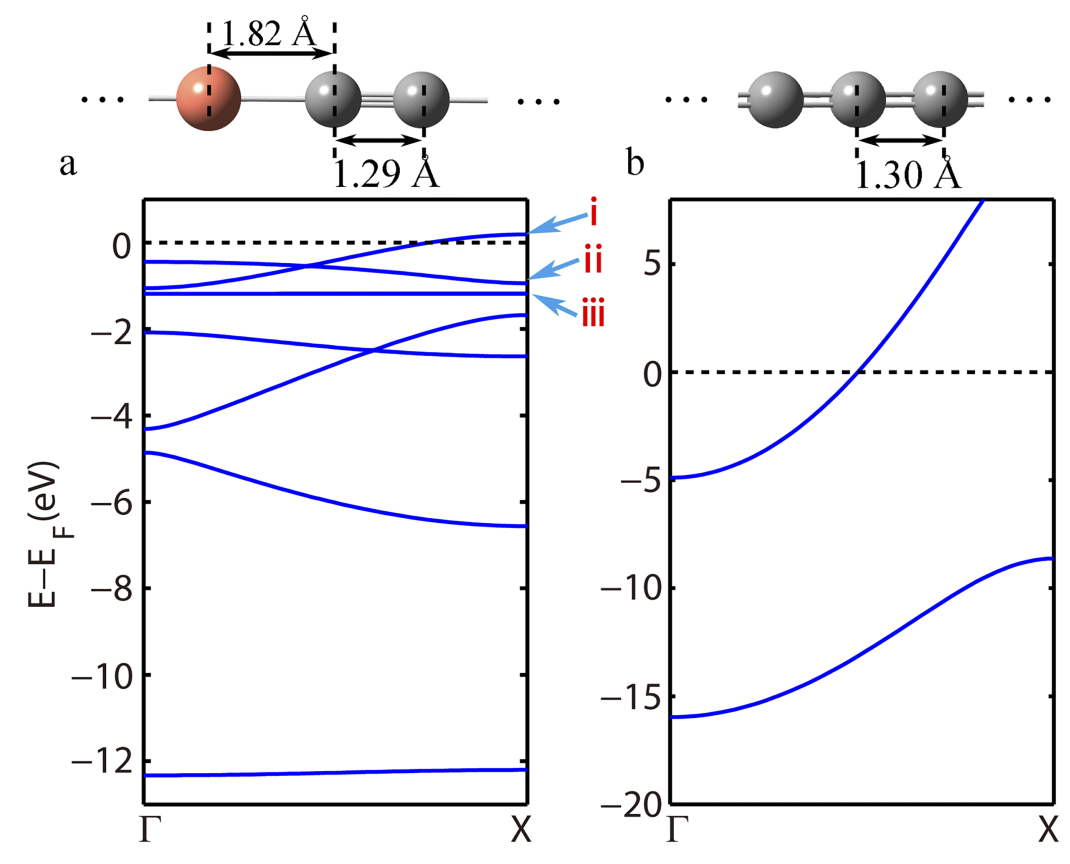

FIG. 1. The atomic and band structures of infinite freestanding $\mathrm{Cu}$-metalated carbyne (a) and clean carbyne in the cumulenic form (b). 
energy bands across the Fermi level, which are, respectively, composed of the carbon $2 p_{x}$ and $2 p_{y}$ atomic orbitals. Since a carbon atom provides 4 valence electrons, the two $\pi$-type energy bands are half-filled. Thus, the clean cumulene chain is prone to Peierls distortion, which results in dimerization. When one $\mathrm{Cu}$ atom per unit cell is incorporated, there are still two degenerate $\pi$-type energy bands intersecting the Fermi level. One is composed of the $\mathrm{C} 2 \mathrm{p}_{\mathrm{x}}$ and $\mathrm{Cu} 3 \mathrm{~d}_{\mathrm{xz}}$ atomic orbitals and the other of $\mathrm{C} 2 \mathrm{p}_{\mathrm{y}}$ and $\mathrm{Cu} 3 \mathrm{~d}_{\mathrm{yz}}$, the corresponding wavefunctions calculated at the $\Gamma$ point are shown in Fig. 2(a). However, the Fermi level cuts across these two energy bands at the k-point $0.75 \pi / \mathrm{a}$. As a result, the $\mathrm{Cu}$-metalated carbyne chain is more robust against distortion than the clean cumulene one due to the much enlarged cell needed to split the bands at or near the edge of the Fermi distribution. ${ }^{33}$ Then it can be expected that its low-bias conductance can reach $2 \mathrm{G}_{0}\left(\mathrm{G}_{0}=2 \times 10^{2} / \mathrm{h}\right.$ is the conductance quantum) when contacted with appropriate electrodes. Considering that the local density of states (LDOS) of the $\mathrm{Cu}$ atom below and around the Fermi level is dominated by the $\mathrm{Cu} 3 \mathrm{~d}$ atomic orbitals (Fig. 2(d)), charge transfer from $\mathrm{Cu}$ to $\mathrm{C}$ occurs in the $\mathrm{Cu}$-metalated carbyne chain. This is corroborated by the population analysis. The Mulliken charges of the $\mathrm{Cu}$ and $\mathrm{C}$ atoms are calculated to be $0.42 \mathrm{e}$ and $-0.21 \mathrm{e}\left(\mathrm{e}=1.60 \times 10^{-19} \mathrm{C}\right)$, respectively. The electronic state of $\mathrm{Cu}$ in carbyne is thus considerably different from that in bulk copper and in the clean $\mathrm{Cu}$ monatomic chain. ${ }^{34}$ Compared with that of bulk copper, the d-bands in the $\mathrm{Cu}$ monatomic chain are shifted upwards but are still below the Fermi level. However, in the $\mathrm{Cu}$-metalated carbyne chain, the energy bands contributed by the $\mathrm{Cu} 3 \mathrm{~d}_{\mathrm{xz}}$ and $3 \mathrm{~d}_{\mathrm{yz}}$ atomic orbitals extend to $0.19 \mathrm{eV}$ above the Fermi level.

Then we move to investigate the electronic transport properties of finite-length chains. As shown in Fig. 3(a), one $\mathrm{Cu}$-metalated carbyne chain composed of 6 carbon and two copper atoms is sandwiched between two (111)-oriented $\mathrm{Cu}$ electrodes through a 4-atom copper cluster arranged in a pyramid configuration at each side. The bond length between the apex $\mathrm{Cu}$ atom and the binding $\mathrm{C}$ one is optimized to be
$1.86 \AA$ A. A sharp transmission peak centered at the Fermi level appears in the equilibrium transmission spectrum [Fig. 3(b)], with the peak value of 1.88 approaching the maximum value of two. Around the Fermi level there are still four more transmission peaks centered at $-0.41 \mathrm{eV},-0.46 \mathrm{eV},-0.66 \mathrm{eV}$, and $-0.79 \mathrm{eV}$ (energies are taken from $E_{\mathrm{F}}=0$ ). By looking at the LDOS projected onto the atomic orbitals of the apex $\mathrm{Cu}$ atoms and of $\mathrm{C}$ in the chain, we can conclude that the transmission peaks centered at $E_{\mathrm{F}},-0.41 \mathrm{eV}$ and $-0.79 \mathrm{eV}$, are dominated by the $\mathrm{Cu} 3 \mathrm{~d}_{\mathrm{xz}} / 3 \mathrm{~d}_{\mathrm{yz}}, 4 \mathrm{p}_{\mathrm{x}} / 4 \mathrm{p}_{\mathrm{y}}$, and $\mathrm{C} 2 \mathrm{p}_{\mathrm{x}} / 2 \mathrm{p}_{\mathrm{y}}$ atomic orbitals, respectively. In contrast the transmission peaks centered at $-0.46 \mathrm{eV}$ and $-0.66 \mathrm{eV}$ are mainly contributed by the $\mathrm{Cu} 3 d_{z^{2}}$, $4 p_{z}$, and $C 2 p_{z}$ atomic orbitals. Because the microscopic configuration of the interface between the $\mathrm{Cu}$ electrode and the $\mathrm{Cu}$-metalated carbyne chain may affect the junction transport significantly, we construct another junction model in which the $\mathrm{Cu}$-metalated carbyne chain binds to the $\mathrm{Cu}$ electrode through one $\mathrm{Cu}$ adatom (see Figure $\mathrm{S} 1$ in the supplementary material). As we can see, the overall shape of the equilibrium transmission spectra of these two junction models is very similar in the energy range from $-1 \mathrm{eV}$ to $1 \mathrm{eV}$. To be specific, they share almost the same energy positions of the four transmission peaks below the Fermi level and the same peak value of 1.88 at the Fermi level. This indicates that the low-bias electronic transport properties of the $\mathrm{Cu}$-metalated carbyne chain connected with the $\mathrm{Cu}$ electrodes have no obvious selectivity on the binding sites of the $\mathrm{Cu}(111)$ surface.

The sharp peaks appearing in the equilibrium transmission spectra and the LDOS around the Fermi level indicate that the electronic coupling between the $\mathrm{Cu}$-metalated carbyne chain and the bulk $\mathrm{Cu}$ electrodes is not very strong. This is not a surprising result. Because $\mathrm{Cu}$ is a noble metal, its energy bands around the Fermi level are dominated by the $\mathrm{Cu}$ $4 \mathrm{~s}$ atomic orbital. ${ }^{35}$ In contrast, the energy bands of the $\mathrm{Cu}$ metalated carbyne chain around the Fermi level are $\pi$-type. This is also confirmed by the calculated $I-V$ characteristics of the junction. As shown in Fig. 3(f), the electric current through the junction exhibits a noticeable saturation when the bias voltage exceeds $0.2 \mathrm{~V}$, namely, when the contribution

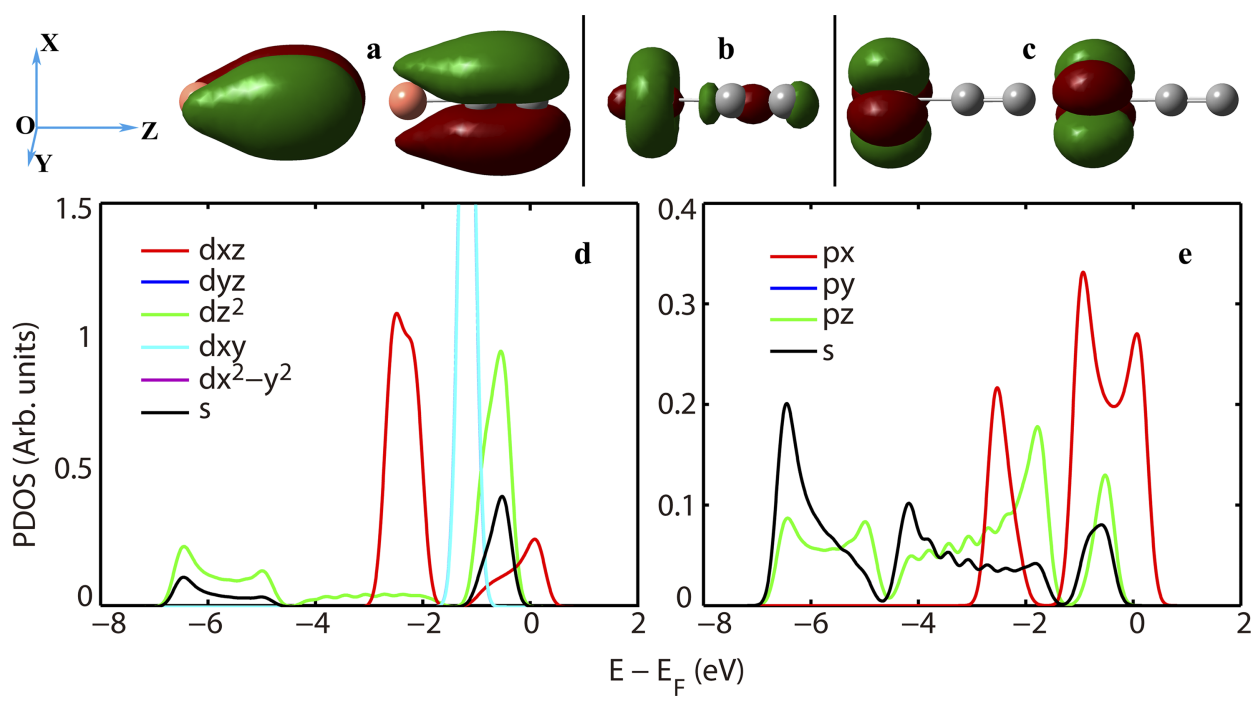

FIG. 2. The wavefunctions ((a)-(c)) of the infinite $\mathrm{Cu}$-metalated carbyne calculated at the $\Gamma$ point for the three energy bands around the Fermi level (labelled i-iii in Fig. 1(a)) and the LDOS projected onto the $\mathrm{Cu} 3 \mathrm{~d} / 4 \mathrm{~s}$ (d) and $\mathrm{C} 2 \mathrm{~s} / 2 \mathrm{p}$ (e) atomic orbitals. Due to the high symmetry, the plots of LDOS for the $\mathrm{Cu}$ $3 \mathrm{~d}_{\mathrm{xz}}$ and $3 \mathrm{~d}_{\mathrm{yz}}, 3 \mathrm{~d}_{\mathrm{xy}}$, and $3 d_{x^{2}-y^{2}}$ atomic orbitals in (d) and those for the C $2 p_{x}$ and $2 p_{y}$ atomic orbitals in (e) overlap with each other. 

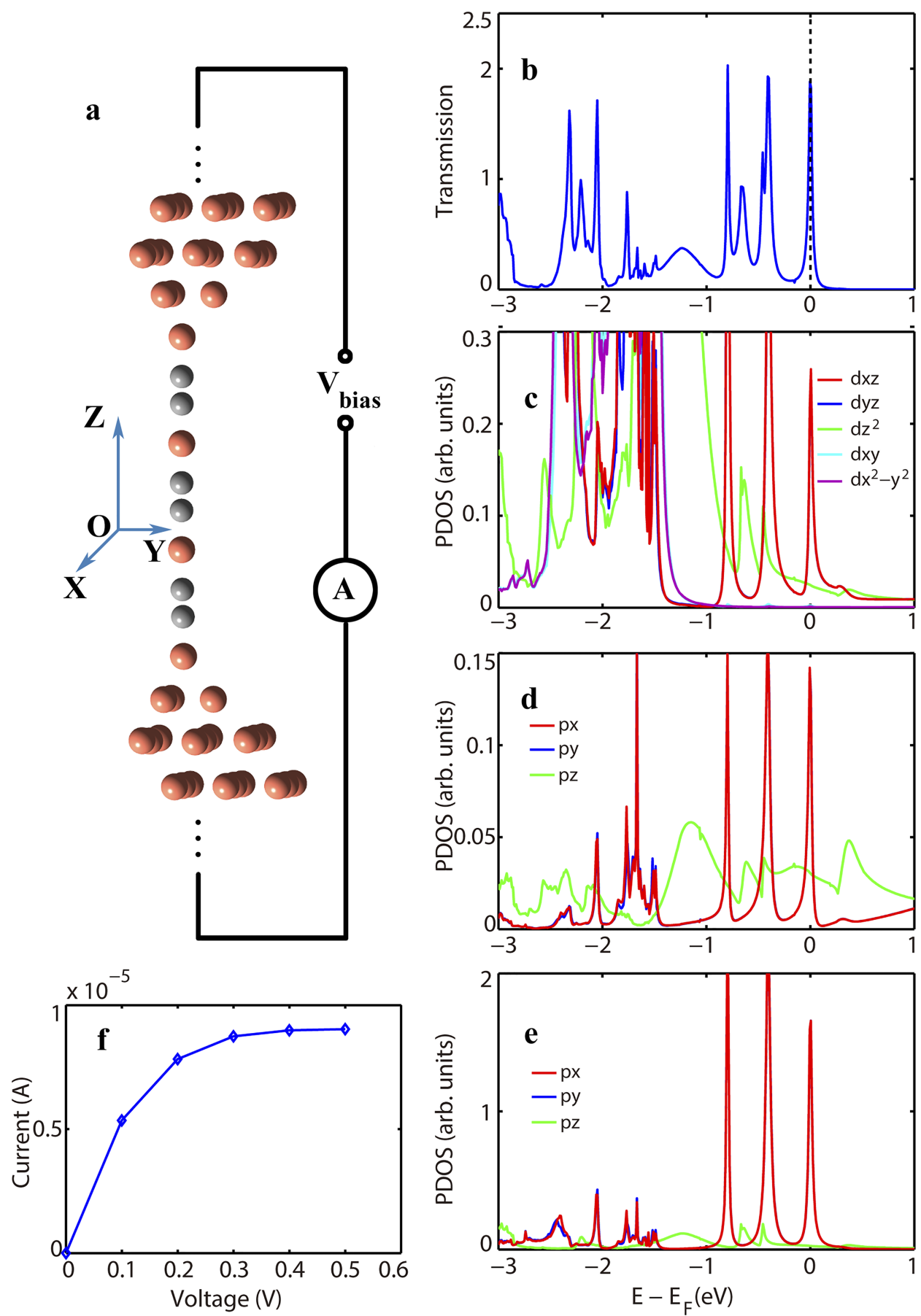

FIG. 3. (a) The optimized atomic structure of the $\mathrm{Cu}$-metalated carbyne chain sandwiched between two $\mathrm{Cu}$ electrodes through a pyramidal $\mathrm{Cu}$ cluster at each side; the equilibrium transmission spectrum (b) and LDOS projected onto the $3 d$ (c) and $4 p(d)$ orbitals of the apex $\mathrm{Cu}$ atom and the $2 \mathrm{p}$ orbitals of the bonding $\mathrm{C}$ atom (e); and the calculated $I-V$ curve (f). Due to the high symmetry, the plots of the $\mathrm{Cu} 3 \mathrm{~d}_{\mathrm{xz}}$ and $3 \mathrm{~d}_{\mathrm{yz}}, 3 \mathrm{~d}_{\mathrm{xy}}$ and $3 d_{x^{2}-y^{2}}$ atomic orbitals in (c), those of the $\mathrm{Cu} 4 \mathrm{p}_{\mathrm{x}}$ and $4 \mathrm{p}_{\mathrm{y}}$ atomic orbitals in (d), and those of the $\mathrm{C} 2 \mathrm{p}_{\mathrm{x}}$ and $2 \mathrm{p}_{\mathrm{y}}$ atomic orbitals in (e) overlap with each other. of the transmission peak at the Fermi level has been fully taken into account.

With the intention of achieving better contacts and thus exploring fully the low-bias transport properties of $\mathrm{Cu}$ metalated carbyne chains, we replace the (111)-oriented $\mathrm{Cu}$ electrodes with (111)-oriented Pt ones. This is because bulk platinum is a transition metal and its energy bands around the Fermi level are dominated by the Pt $5 \mathrm{~d}$ atomic orbitals. ${ }^{35}$ It has also been shown experimentally that strong $\mathrm{Pt}-\mathrm{C}$ bonds can be formed at the molecule-Pt interfaces and independent $\mathrm{Pt}$ atoms can act as nucleation sites for the formation of carbyne chains. ${ }^{36,37}$ As shown in Fig. 4(a), the Cu-metalated carbyne chain composed of six carbon atoms and two copper atoms is assumed to bind at the atop site of the $\operatorname{Pt}(111)$ surface, and the
$\mathrm{Pt}-\mathrm{C}$ bond length is optimized to be $1.92 \AA$. Just as expected, the equilibrium transmission spectrum shows a plateau with the value greater than unity in the energy range from $-0.85 \mathrm{eV}$ to $0.05 \mathrm{eV}$, superimposed with five sharp peaks. By comparing the LDOS of the binding $\mathrm{Pt}$ and $\mathrm{C}$ atoms and that of $\mathrm{Cu}$ atoms in the metalated carbyne chain, we can conclude that the transmission around the Fermi level is mainly contributed by the $\mathrm{Pt}$ $5 \mathrm{~d}_{\mathrm{xz}} / 5 \mathrm{~d}_{\mathrm{yz}}, \mathrm{C} 2 \mathrm{p}_{\mathrm{x}} / 2 \mathrm{p}_{\mathrm{y}}$, and $\mathrm{Cu} 3 \mathrm{~d}_{\mathrm{xz}} / 3 \mathrm{~d}_{\mathrm{yz}}$ atomic orbitals. The transmission peaks centered at $\mathrm{E}_{F},-0.32 \mathrm{eV}$ and $-0.68 \mathrm{eV}$, are dominated by the Pt $5 \mathrm{~d}_{\mathrm{xz}} / 5 \mathrm{~d}_{\mathrm{yz}}, \mathrm{C} 2 \mathrm{p}_{\mathrm{x}} / 2 \mathrm{p}_{\mathrm{y}}$, and $\mathrm{Cu} 3 \mathrm{~d}_{\mathrm{xz}} / 3 \mathrm{~d}_{\mathrm{yz}}$ atomic orbitals, while those at $-0.54 \mathrm{eV}$ and $-0.80 \mathrm{eV}$ originate from $\mathrm{Pt} 5 d_{z^{2}}, \mathrm{C} 2 \mathrm{p}_{\mathrm{z}}$, and $\mathrm{Cu} 3 d_{z^{2}}$. Furthermore, the transmission coefficient at $\mathrm{E}_{F}$ is calculated to 1.81 , and the transmission coefficient minimum in the plateau is still as high 


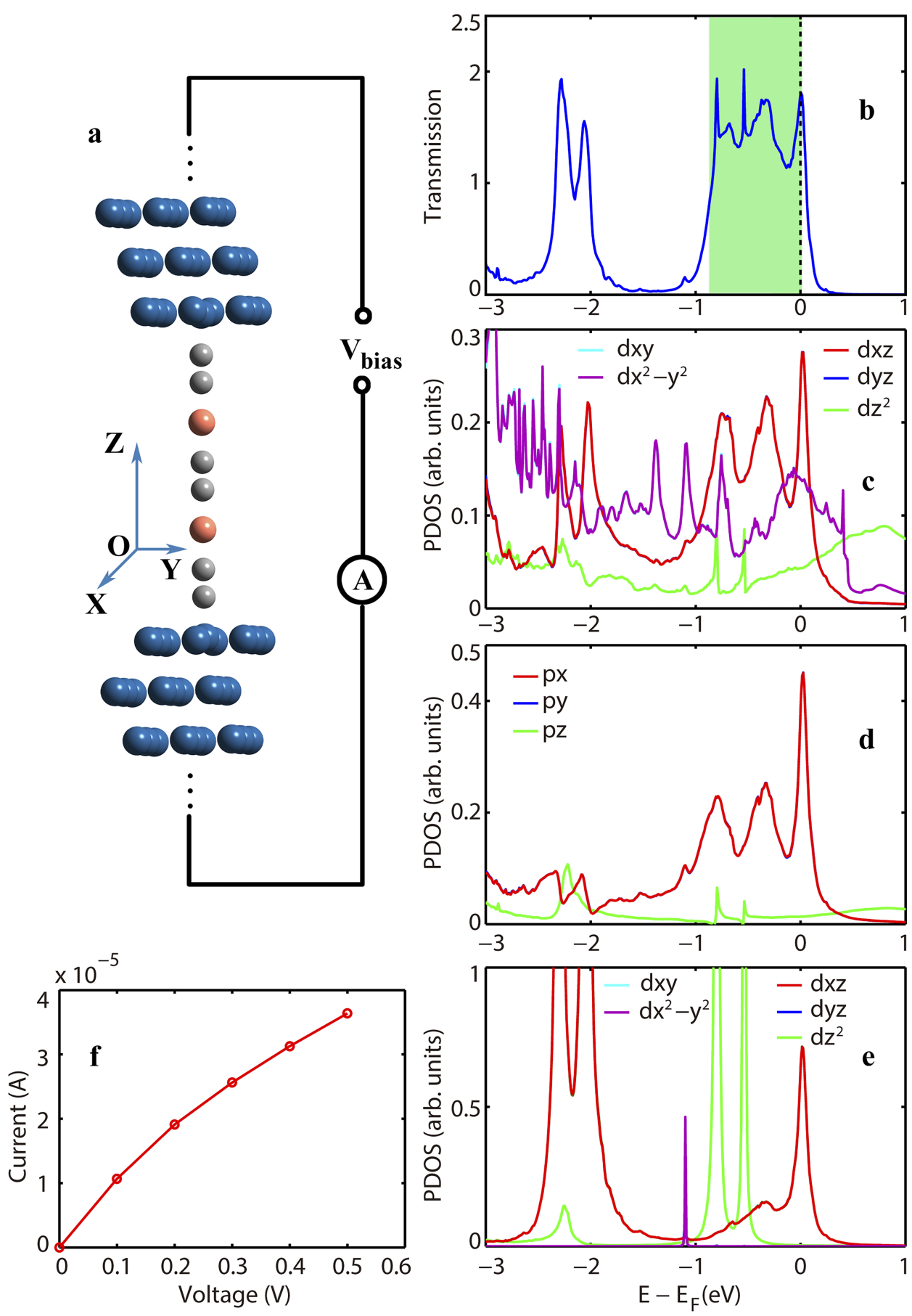

FIG. 4. (a) The optimized atomic structure of the $\mathrm{Cu}$-metalated carbyne chain connected to two Pt electrodes at the atop sites; the equilibrium transmission spectrum (b) and LDOS projected onto the $5 d$ orbitals of the bonding Pt atom (c), the $2 p$ orbitals of the bonding $C$ atom (d) and the $3 \mathrm{~d}$ orbitals of the $\mathrm{Cu}$ atom (e); and the calculated $I-V$ curve (f). Due to the high symmetry, the plots of the Pt $5 \mathrm{~d}_{\mathrm{xz}}$ and $5 \mathrm{~d}_{\mathrm{yz}}, 5 \mathrm{~d}_{\mathrm{xy}}$ and $5 d_{x^{2}-y^{2}}$ atomic orbitals in (c), those of the $C 2 p_{x}$ and $2 p_{y}$ atomic orbitals in (d), and those of the $\mathrm{Cu} 3 \mathrm{~d}_{\mathrm{xz}}$ and $3 d_{y z}, 3 d_{x y}$ and $3 d_{x^{2}-y^{2}}$ in (e) overlap with each other.

as 1.14 (this value is taken in a transmission valley located at $-0.13 \mathrm{eV}$ ). Thus, due to the much stronger electronic coupling between the Pt $5 \mathrm{~d}$ and the $\mathrm{C} 2 \mathrm{p}$ atomic orbitals, Pt appears as a more appropriate electrode material to reveal the intrinsic $2 \mathrm{G}_{0}$ conductance of $\mathrm{Cu}$-metalated carbyne chains. We also calculate the $I-V$ curve of the $\mathrm{Cu}$-metalated carbyne chain connected with Pt electrodes [see Fig. 4(f)]. Clearly, the $I-V$ curve shows a linear dependence at low bias voltages, and the current values are also greater than that with the $\mathrm{Cu}$ electrodes [Fig. 3(f)].

In order to investigate the effects of the binding configuration on the transport properties of $\mathrm{Cu}$-metalated carbyne chains connected with Pt electrodes, we have inserted one $\mathrm{Pt}$ adatom between the $\operatorname{Pt}(111)$ surface and the chain (see
Figure S2 in the supplementary material). It can be seen from the equilibrium transmission spectrum displayed in Fig. S2(b) that, although the two transmission peaks dominated by the $\mathrm{Pt}$ $5 d_{z^{2}}$ and $C 2 p_{z}$ atomic orbitals are still positioned at $-0.54 \mathrm{eV}$ and $-0.80 \mathrm{eV}$, significant changes occur for the transmission peaks assigned to the $\mathrm{Pt} 5 \mathrm{~d}_{\mathrm{xz}} / 5 \mathrm{~d}_{\mathrm{yz}}, \mathrm{C} 2 \mathrm{p}_{\mathrm{x}} / 2 \mathrm{p}_{\mathrm{y}}$, and $\mathrm{Cu} 3 \mathrm{~d}_{\mathrm{xz}} / 3 \mathrm{~d}_{\mathrm{yz}}$ atomic orbitals. In details, the transmission peak appearing at $-0.68 \mathrm{eV}$ for the atop site completely disappears for the adatom site, the one centered at $-0.32 \mathrm{eV}$ for the atop site shifts to $-0.21 \mathrm{eV}$ for the adatom one, the one at $\mathrm{E}_{F}$ for the atop site becomes a much broadened shoulder for the adatom site and the transmission coefficient at the Fermi level is also reduced to 1.22 . However, the calculated $I-V$ curves for these two junction models show a much similar behavior because the electric 
current through the junction is an integral of the transmission spectrum in the bias window. Therefore, the strong electronic coupling between the $\mathrm{Cu}$-metalated carbyne chain and the $\mathrm{Pt}$ electrodes manifests the excellent conducting ability of $\mathrm{Cu}$ metalated carbyne chains though the binding configurations do somewhat mediate their interactions.

\section{CONCLUSION}

We have investigated the atomic structure and electronic transport properties of $\mathrm{Cu}$-metalated carbyne monatomic chains using the NEGF + DFT approach. Our calculations show that, in contrast to clean carbyne that is prone to the Peierls distortion and thus can be a metal or a semiconductor depending on the application conditions, $\mathrm{Cu}$-metalated carbyne always preserves its metallic character due to charge transfer between the incorporated $\mathrm{Cu}$ atom and the neighboring carbon atoms. Since two degenerate $\pi$-type energy bands intersect the Fermi level, $\mathrm{Cu}$-metalated carbyne can act as highly conductive molecular wires. This is demonstrated with a finite $\mathrm{Cu}$-metalated carbyne chain connected to two (111)-oriented Pt electrodes, for which a broad transmission plateau appears around the Fermi level and nearly linear $I-V$ curves are obtained for both the atop and adatom binding sites. These findings are helpful in facilitating the applications of carbyne in future nano/molecular electronics.

\section{SUPPLEMENTARY MATERIAL}

See supplementary material for the atomic structures and electronic transport properties of a finite $\mathrm{Cu}$-metalated carbyne chain connected to (111)-oriented $\mathrm{Cu}$ and $\mathrm{Pt}$ electrodes at the adatom site.

\section{ACKNOWLEDGMENTS}

This project was supported by the National Natural Science Foundation of China (Grant Nos. 61621061, 61271050, and 61671021), the MOST of China (Grant No. 2013CB933404), and the National Key Research and Development Program (Grant No. 2016YFA0201901). S.S. thanks additional funding support from the European Research Council (QUEST project) and by AMBER (Grant No. 12/RC/2278).

${ }^{1}$ A. D. Franklin, Science 349, 704 (2015).

${ }^{2}$ D.-M. Sun, C. Liu, W.-C. Ren, and H.-M. Cheng, Small 9, 1188 (2013).

${ }^{3}$ C. Wang, K. Takei, T. Takahashi, and A. Javey, Chem. Soc. Rev. 42, 2592 (2013).
${ }^{4}$ S. Park, M. Vosguerichian, and Z. Bao, Nanoscale 5, 1727 (2013).

${ }^{5}$ R. B. Heimann, S. E. Evsyukov, and L. Kavan, Carbyne and Carbynoid Structures (Kluwer Academic Publishers, 1999).

${ }^{6}$ R. H. Baughman, Science 312, 1009 (2006).

${ }^{7}$ W. A. Chalifoux and R. R. Tykwinski, Nat. Chem. 2, 967 (2010).

${ }^{8}$ L. Shi, P. Rohringer, K. Suenaga, Y. Niimi, J. Kotakoshi, J. C. Meyer, H. Peterlik, M. Wanko, S. Cahangirov, A. Rubio, Z. J. Lapin, L. Novotny, P. Ayala, and T. Pichler, Nat. Mater. 15, 634 (2016).

${ }^{9}$ C. S. Casari, M. Tommasini, R. R. Tykwinski, and A. Milani, Nanoscale 8, 4414 (2016).

${ }^{10}$ F. Banhart, Beilstein J. Nanotechnol. 6, 559 (2015).

${ }^{11}$ P. Bonardi, S. Achilli, G. F. Tantardini, and R. Martinazzo, Phys. Chem. Chem. Phys. 17, 18413 (2015).

${ }^{12}$ V. I. Artyukhov, M. Liu, and B. I. Yakobson, Nano Lett. 14, 4224 (2014).

${ }^{13}$ C. Neiss, E. Trushin, and A. Görling, ChemPhysChem 15, 2497 (2014).

${ }^{14}$ O. Cretu, A. R. Botello-Mendez, I. Janowska, C. Pham-Huu, J.-C. Charlier, and F. Banhart, Nano Lett. 13, 3487 (2013).

${ }^{15}$ A. La Torre, A. Botello-Mendez, W. Baaziz, J.-C. Charlier, and F. Banhart, Nat. Commun. 6, 6636 (2015).

${ }^{16}$ Q. Sun, L. Cai, S. Wang, R. Widmer, H. Ju, J. Zhu, L. Li, Y. He, P. Ruffieux, R. Fasel, and W. Xu, J. Am. Chem. Soc. 138, 1106 (2016).

${ }^{17}$ H. F. Wittmann, R. H. Friend, M. S. Khan, and J. Lewis, J. Chem. Phys. 101, 2693 (1994).

${ }^{18}$ D. Beljonne, H. F. Wittmann, A. Köhler, S. Graham, M. Younus, J. Lewis, P. R. Raithby, M. S. Khan, R. H. Friend, and J. L. Brédas, J. Chem. Phys. 105, 3868 (1996).

${ }^{19}$ M. Springborg and R. C. Albers, Phys. Rev. B 53, 10626 (1996).

${ }^{20}$ Y. Meir and N. S. Wingreen, Phys. Rev. Lett. 68, 2512 (1992).

${ }^{21}$ P. Hohenberg and W. Kohn, Phys. Rev. 136, B864 (1964).

${ }^{22}$ W. Kohn and L. J. Sham, Phys. Rev. 140, A1133 (1965).

${ }^{23}$ Y. Xue, S. Datta, and M. A. Ratner, Chem. Phys. 281, 151 (2002).

${ }^{24}$ M. Brandbyge, J.-L. Mozos, P. Ordejón, J. Taylor, and K. Stokbro, Phys. Rev. B 65, 165401 (2002).

${ }^{25}$ J. Zhang, S. Hou, R. Li, Z. Qian, R. Han, Z. Shen, X. Zhao, and Z. Xue, Nanotechnology 16, 3057 (2005).

${ }^{26}$ R. Li, J. Zhang, S. Hou, Z. Qian, Z. Shen, X. Zhao, and Z. Xue, Chem. Phys. 336, 127 (2007).

${ }^{27}$ A. R. Rocha, V. M. García-Suárez, S. W. Bailey, C. J. Lambert, J. Ferrer, and S. Sanvito, Nat. Mater. 4, 335 (2005).

${ }^{28}$ A. R. Rocha, V. M. García-Suárez, S. Bailey, C. Lambert, J. Ferrer, and S. Sanvito, Phys. Rev. B 73, 085414 (2006).

${ }^{29}$ I. Rungger and S. Sanvito, Phys. Rev. B 78, 035407 (2008).

${ }^{30}$ J. M. Soler, E. Artacho, J. D. Gale, A. García, J. Junquera, P. Ordejón, and D. Sánchez-Portal, J. Phys.: Condens. Matter 14, 2745 (2002).

${ }^{31}$ N. Troullier and J. L. Martins, Phys. Rev. B 43, 1993 (1991).

${ }^{32}$ J. P. Perdew, K. Burke, and M. Ernzerhof, Phys. Rev. Lett. 77, 3865 (1996).

${ }^{33}$ R. E. Peierls, Quantum Theory of Solids (Oxford University Press, 1955).

${ }^{34}$ E. Santos, P. Quaino, G. Soldano, and W. Schmickler, Electrochem. Commun. 11, 1764 (2009).

${ }^{35}$ D. A. Papaconstantopoulos, Handbook of the Band Structure of Elemental Solids: From $Z=1$ to $Z=112,2$ nd ed. (Springer, New York, 2015).

${ }^{36}$ Y. Fu, S. Chen, A. Kuzume, A. Rudnev, C. Huang, V. Kaliginedi, M. Baghernejad, W. Hong, T. Wandlowski, S. Decurtins, and S. Liu, Nat. Commun. 6, 6403 (2015).

${ }^{37}$ E. Kano, M. Takeguchi, J.-I. Fujita, and A. Hashimoto, Carbon 80, 382 (2014). 